\title{
Les règles d'urbanisme servent-elles le développement urbain? Réflexions à partir d'un exemple canadien
}

\section{Jean Ruegg, Lausanne}

\section{Enjeux urbanistiques des zones industrielles centrales}

A Toronto - tout comme d'ailleurs dans d'autres villes américaines - plusieurs zones industrielles ont une valeur stratégique considérable en raison de leur proximité immédiate au centre des affaires. Elles constituent même, depuis les années quatrevingts, une sorte d'anachronisme d'autant plus étonnant que la pression immobilière liée aux activités présentes dans la CBD (Central business district) est considérable, malgré la crise immobilière du début des années quatre-vingt-dix et l'émergence de nouveaux centres de service, articulés en réseau et implantés à la périphérie de l'agglomération de Toronto (GARREAU 1992; FRISKEN 1988).

Il est intéressant, dans cette perspective, d'examiner comment les autorités de Toronto utilisent les règles d'urbanisme pour composer avec cette évolution liée essentiellement aux forces économiques du marché. Les arguments développés dans cet article s'appuient sur des documents élaborés par le Service de l'urbanisme de la ville de Toronto (City of Toronto 1996) et sur des recherches personnelles menées dans le cadre d'un travail de doctorat consacré au zonage (RUEGG 1997, 1992 et 1989). Ils sont articulés en quatre parties. La politique urbaine en vigueur dans le quartier de KingSpadina est d'abord présentée. Puis les phénomènes de restructuration qui sont à l'œuvre tant au sein du secteur de la mode (c'est la principale activité industrielle localisée dans le quartier) qu'à la périphérie immédiate de King-Spadina sont rapidement évoqués. Ces phénomènes ont des conséquences territoriales importantes. Ils obligent les autorités locales à reprendre régulièrement les règles d'urbanisme qu'elles ont édictées pour préserver la diversité des emplois industriels au centre de Toronto. L'énoncé de ces ajustements fait l'objet de la troisième partie. Quant à la conclusion, elle porte sur les limites d'une telle démarche. Les actions développées par l'acteur public ont trois caractéristiques principales. Elles tendent à être réactives plutôt qu'anticipatrices, elles n'intègrent pas la problématique foncière et elles sont souvent modifiées. Leur mise en œuvre annule donc systématiquement l'effet stabilisateur que le zonage exerce sur les prix fonciers lorsqu'il est appliqué durablement. Il n'est alors pas exagéré de prétendre que dans un tel contexte les règles d'urbanisme se révèlent insuffisantes pour agir sur le développement urbain.

\section{Politique urbaine pour King-Spadina}

La zone industrielle de King-Spadina (fig. 1) couvre environ 90 hectares. Elle abrite, avec la zone de King-Parliament, les activités qui, dès la fin du XIXe siècle,jouent un rôle essentiel pour structurer la ville et pour alimenter l'essor démographique et économique de la cité. L'industrie devient l'utilisation dominante à King-Spadina dès le début du siècle, en liaison avec la réalisation du chemin de fer. Sur le plan de la morphologie urbaine, sa présence est marquée par le plan en damier et par le développement d'une architecture industrielle de qualité qui est élevée aujourd'hui au rang de patrimoine historique digne de protection (GREenBerg \& LEWINBERG 1996; Miller 1996).

En 1985, l'industrie du vêtement constitue encore l'activité principale du quartier. Elle rassemble $60 \%$ du total des emplois offerts à King-Spadina. Et puis surtout, elle sert de référence et de point d'appui pour légitimer, pour forger, et pour évaluer l'efficacité de la politique de soutien à l'industrie que les autorités locales mettent en place dès les années soixante-dix. Cette politique s'inscrit dans une stratégie globale. Il s'agit de maintenir une composante industrielle au centre de la ville afin de préserver une bonne diversité des emplois localisés dans les secteurs les plus facilement accessibles en transport public. Elle repose essentiellement sur deux types d'instruments qui sont plus ou moins explicitement calqués sur les besoins de l'industrie du vêtement:

- Des mesures de zonage dédiées à la régulation de l'utilisation du sol. Mises en place dès les années cinquante, elles sont consignées dans un plan directeur de quartier: le King-Spadina Official Plan Part II qui est élaboré une première fois en 1978, puis révisé en 1980, 1988 et 1996. Elles sont confirmées dans les plans d'affectation des zones (City of Toronto 1996; Miller 1996)

- Des actions sectorielles destinées à soutenir plus spécifiquement l'industrie du vêtement. Elles sont articulées autour de formules de partenariat public-privé initiées ou organisées par le Fashion Industry Liaison Committee (FILC). Ce groupe est créé en 1984. Son mandat consiste 


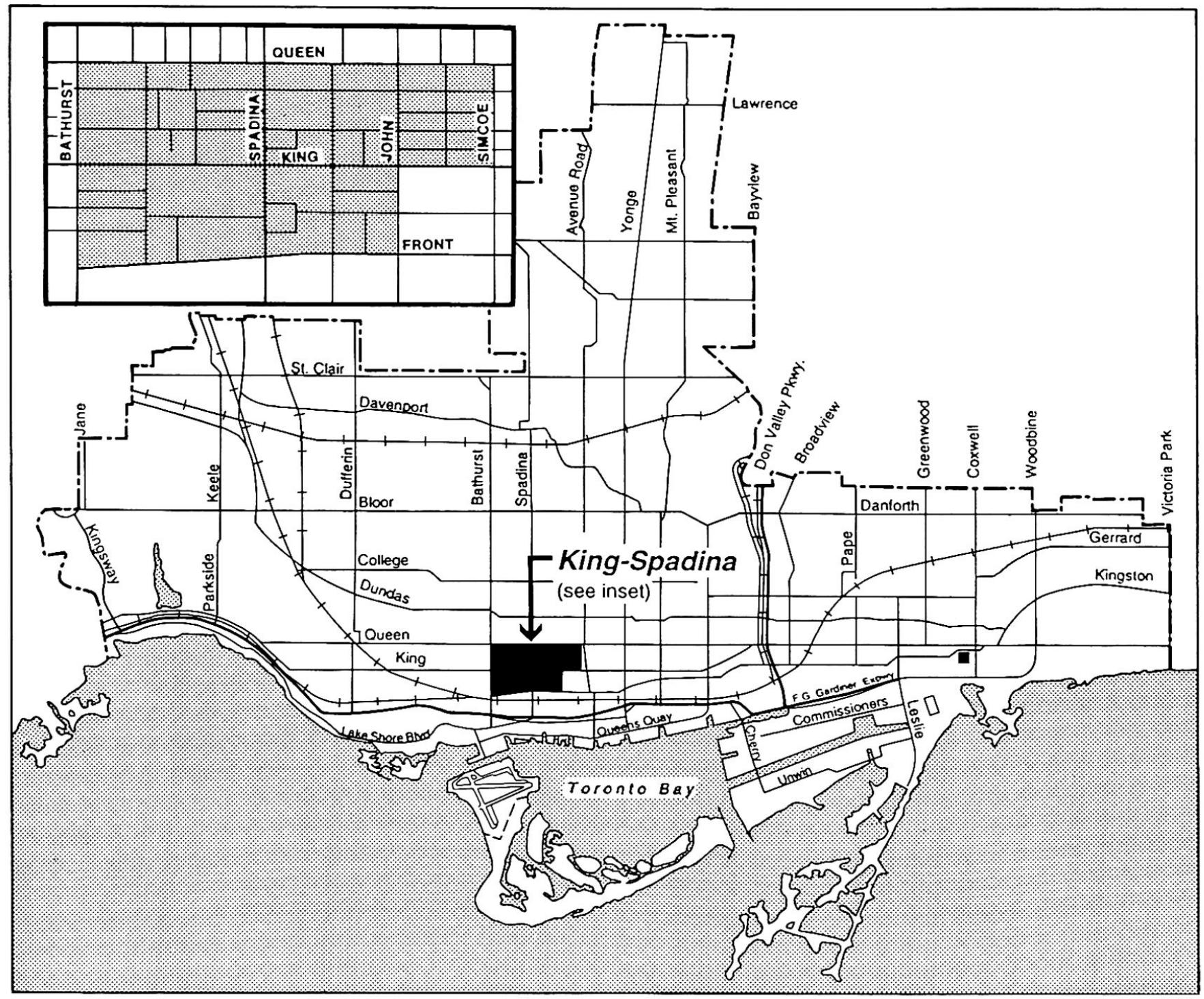

Fig. 1: Localisation du quartier industriel de King-Spadina

Location of the industrial area of King-Spadina

Standort des Industrieviertels King-Spadina

Source: City of ToRonto (1996: 3)

essentiellement à promouvoir l'industrie du vêtement et à fournir des préavis aux autorités locales pour des objets qui concernent directement ce secteur économique. Il rassemble les différents partenaires de la branche (politiciens, représentants des patrons, des syndicats et des responsables de la formation des employés). Il est coordonné par un membre de la division économique du service de l'urbanisme de la ville de Toronto.

L'objectif des autorités de maintenir l'industrie à King-Spadina n'est cependant pas dépourvu d'ambiguïtés. D'abord, ce n'est certainement pas l'unique but qu'elles poursuivent. Elles sont également soucieuses de préserver un patrimoine architectural historiquement lié à la présence de l'industrie à King-Spadina. Ensuite, l'industrie du vêtement, dont l'existence légitime leur action politique, est soumise à de profondes mutations (Gibis 1988; Scotт 1988). Enfin, l'affectation industrielle du quartier de King-Spadina est soumise à la pression des activités de la CBD.

Le cas de King-Spadina est alors propice pour montrer les difficultés auxquelles les autorités doivent faire face dans leur tâche de régulation de l'utilisation du sol. Il permet, plus précisément, d'examiner la pertinence et l'utilité du zonage pour composer avec des changements intervenant dans la durée, dans un contexte où l'institution de la propriété privée du sol n'est pas remise en question (Booth 1989; Christman 1994). 


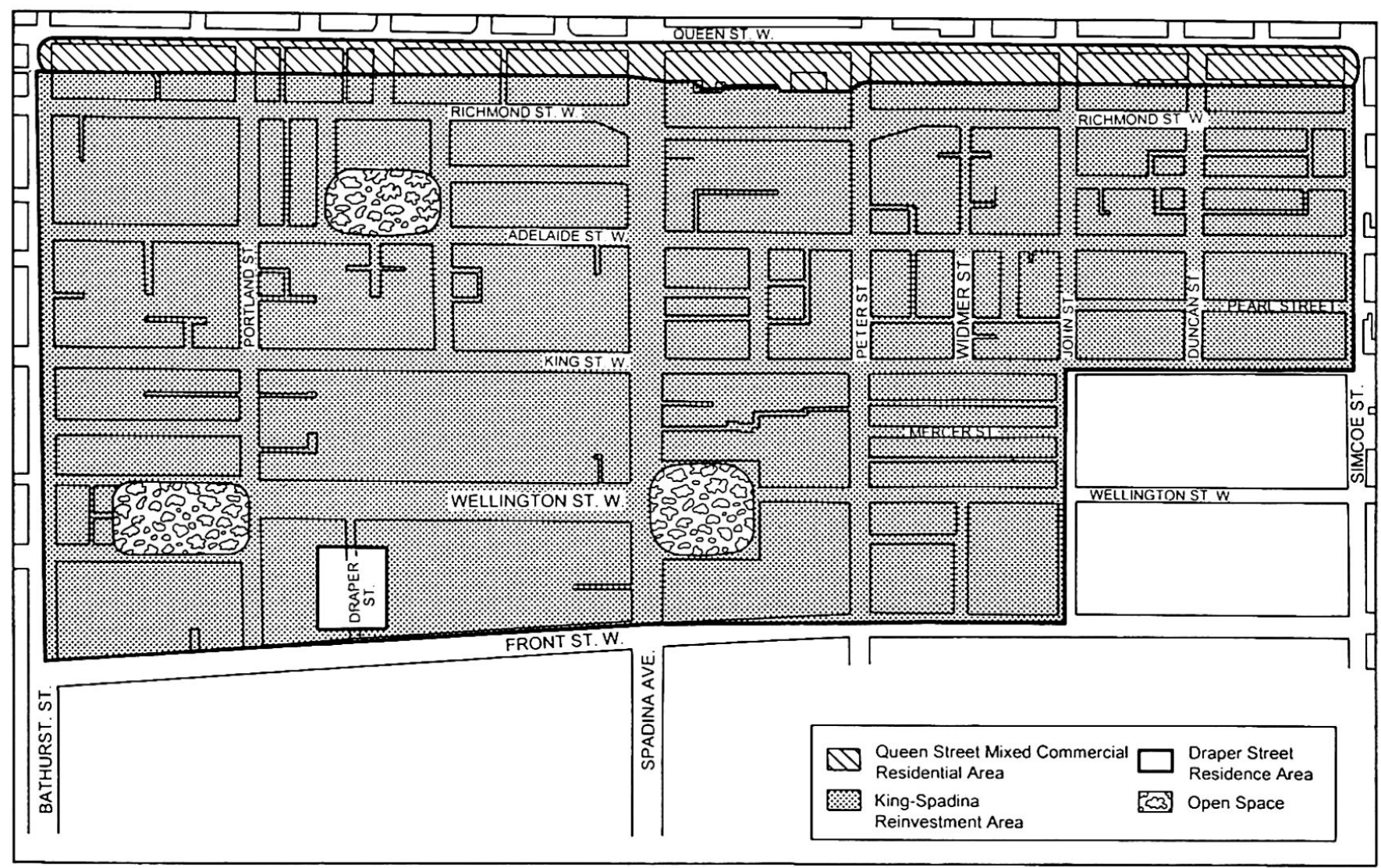

Fig. 2: King-Spadina Official Plan Part II (1988)

King-Spadina Official Plan Part II of 1988

Offizieller Entwicklungsplan Teil II für King-Spadina, 1988

Source: CiTY OF TORONTO (1996: 24)

\section{Pression sur l'activité à King-Spadina}

Cette pression a au moins deux sources qui se renforcent et se complètent et qui toutes deux concourent à modifier le territoire de King-Spadina. La première est liée à des changements qui sont propres à l'industrie de la mode. La seconde résulte des anticipations et des attentes d'acteurs qui spéculent sur une extension de la CBD et des activités qui lui sont associées. La collectivité publique n'a en l'occurrence pas prise ni sur le premier, ni sur le second de ces phénomènes.

\subsection{Pression liée aux changements à l'industrie de la mode}

Les mutations qui se développent au sein de l'industrie du vêtement sont liées à plusieurs évènements qui participent à la fragiliser. Il faut d'abord mettre en exergue l'accroissement de la concurrence en provenance des pays de l'Asie du sud-est qui est encore exacerbée dans les années quatre-vingt-dix avec l'accord de libre-échange passé entre le Canada, les Etats-Unis et le Mexique. Pour composer avec cela, l'industrie du vêtement (garment or apparel industry) subit une recon- version totale. Dans les années quatre-vingts, les entreprises localisées à King-Spadina s'orientent très explicitement vers une industrie de la mode (fashion industry). Or cette évolution marque des changements:

- dans les modalités d'organisation de la production: il s'agit désormais de produire plusieurs collections différentes par saison;

- de clientèle et de positionnement:l'industrie vise dorénavant un «marché niche» réservé à une clientèle aisée;

- de métiers: le design, la gestion, la commercialisation et le marketing gagnent en importance par rapport aux fonctions classiques de production et s'affirment comme des savoir-faire nécessaires au développement de l'industrie de la mode.

Dans ce contexte, la survie de l'industrie de la mode va reposer sur une très forte flexibilité interne (Scotr 1984). Ceci a des conséquences spatiales considérables. A la fin des années quatre-vingts, et même si les indices généraux de l'emploi sont à la baisse, l'industrie de la mode dépend encore fortement de la main d'œuvre. Pour réduire les coûts de 
production au maximum, elle tend alors à se structurer autour d'une multitude de petites firmes qui exercent chacune une fonction spécifique (design, élaboration des patrons, coupe du tissu, assemblage, finition, conditionnement, vente) et qui entretiennent entre elles de fortes relations basées sur la sous-traitance et la disponibilité du système de production. La proximité est alors une condition essentielle. Les entreprises doivent être localisées les unes à côté des autres pour permettre à cette organisation en réseau de fonctionner. Sur ce plan, la convergence d'intérêts est forte entre l'objectif des autorités de préserver une utilisation industrielle à King-Spadina et le besoin de l'industrie de la mode de pouvoir rester sur place et de jouer avec l'effet d'agglomération (Cluster).

Mais bientôt d'autres évènements vont intervenir qui ont plutôt tendance à destructurer ce réseau de petites entreprises. Avec sa nouvelle orientation, l'industrie de la mode voit ses besoins en surface se modifier. Elle va libérer des emplacements à KingSpadina. D'autres acteurs, aux capacités financières souvent supérieures, vont s'établir dans le quartier et contribuer à l'augmentation des loyers. $\mathrm{Ce}$ phénomène va alors se retourner contre l'industrie de la mode. Pour occuper son marché niche, elle requiert en effet davantage de surfaces d'exposition situées à des endroits stratégiques et donc coûteux. Par ailleurs, à la fin des années quatre-vingts, les baux de plusieurs entreprises implantées depuis de nombreuses années viennent à échéance. Ils sont renégociés à la hausse et leur durée est considérablement réduite. Ce dernier phénomène a plusieurs explications. Il est d'abord le produit d'une plus forte spéculation dans le quartier. Ainsi, entre 1976 et 1988 , le taux d'accroissement des loyers est plus élevé pour les surfaces industrielles à King-Spadina que pour les bureaux dans la CBD. Mais il découle surtout du changement de l'identité du propriétaire. Dans les années soixante, celui-ci est souvent actif dans l'industrie du vêtement. Il considère ses biensfonds comme un moyen de production. Il pratique souvent des prix qui servent les intérêts de la branche. Dans les années quatre-vingts en revanche, il n'est plus du tout lié à l'industrie. Il traite ses propriétés comme un objet d'investissement dont il essaie de tirer le meilleur rendement possible indépendamment des besoins ou des spéci-

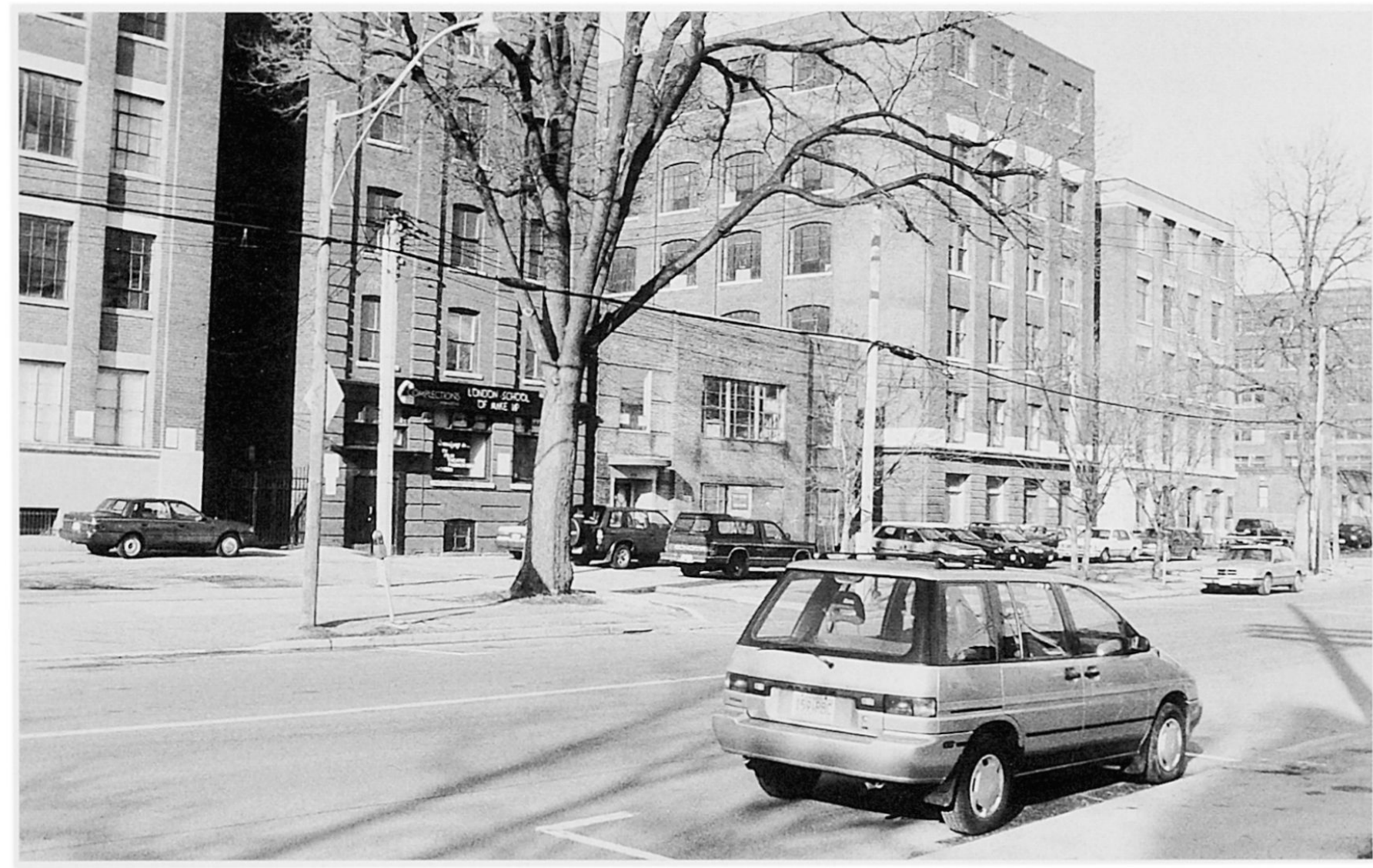

Photo 1: Rue Wellington

Rue Wellington, exemple de redéveloppement souhaité pour King-Spadina: la largeur de la rue et les arbres apportent une qualité environnementale certaine, tandis que les immeubles rénovés abritent des magasins, des écoles, des logements et des ateliers liés à l'industrie de la mode.

J. RuEGG, février 1997 
ficités de ses locataires. Tout ces éléments concourent à augmenter la part des montants que les entreprises doivent consacrer à leurs locaux. Dans ce contexte, soit elles sont assez petites pour louer à bon compte des surfaces industrielles résiduelles, soit elles sont contraintes de réduire d'autres coûts de production tels que les salaires, soit elles font faillites. D'autres stratégies émergent cependant. Certaines firmes qui sont positionnées dans le segment le plus profitable du marché, c'est-à-dire là où le design et la qualité jouent un rôle essentiel, et qui sont assez grosses pour réaliser les investissements requis pour accéder à la production assistée par ordinateur (PAO), tendent à réinternaliser l'ensemble du système de production. Ce faisant, elles s'affranchissent considérablement de toute dépendance quant à leur localisation. Elles décentralisent leur unité de production à la périphérie là où les loyers sont meilleur marché et maintiennent seulement des showrooms à KingSpadina afin de montrer et de vendre leurs collections aux représentants des grands magasins ou des boutiques de modes. Cette tendance, qui se dessinait déjà à la fin des années quatre-vingts, a bien sûr été amplifiée depuis, grâce à la baisse des coûts de la PAO.

Cette évolution est remarquable. Elle marque une rupture importante. Pour la première fois dans l'histoire du quartier, le destin de l'industrie du vêtement et le futur de King-Spadina ne sont plus forcément liés. Cette mutation affaiblit considérablement la politique des autorités de la ville de Toronto quant au maintien et à la défense de l'industrie à King-Spadina.

\subsection{Pression liée à l'extension de la CBD}

L'extension de la CBD, dans les années soixante, interfère peu avec les activités des quartiers voisins. Au contraire, elle les confirme dans la mesure où pratiquement les mêmes intérêts sont présents à la fois dans les entreprises de King-Spadina et de King-Parliament et dans les firmes financières de la CBD. Mais cet arrangement n'est pas durable. Un conflit réel d'utilisation du sol se développe à partir des années quatre-vingts lorsque l'essor de la CBD entre en concurrence avec le maintien de l'industrie. La poussée qu'elle exerce est particulièrement forte dans les zones de lisière où elle borde

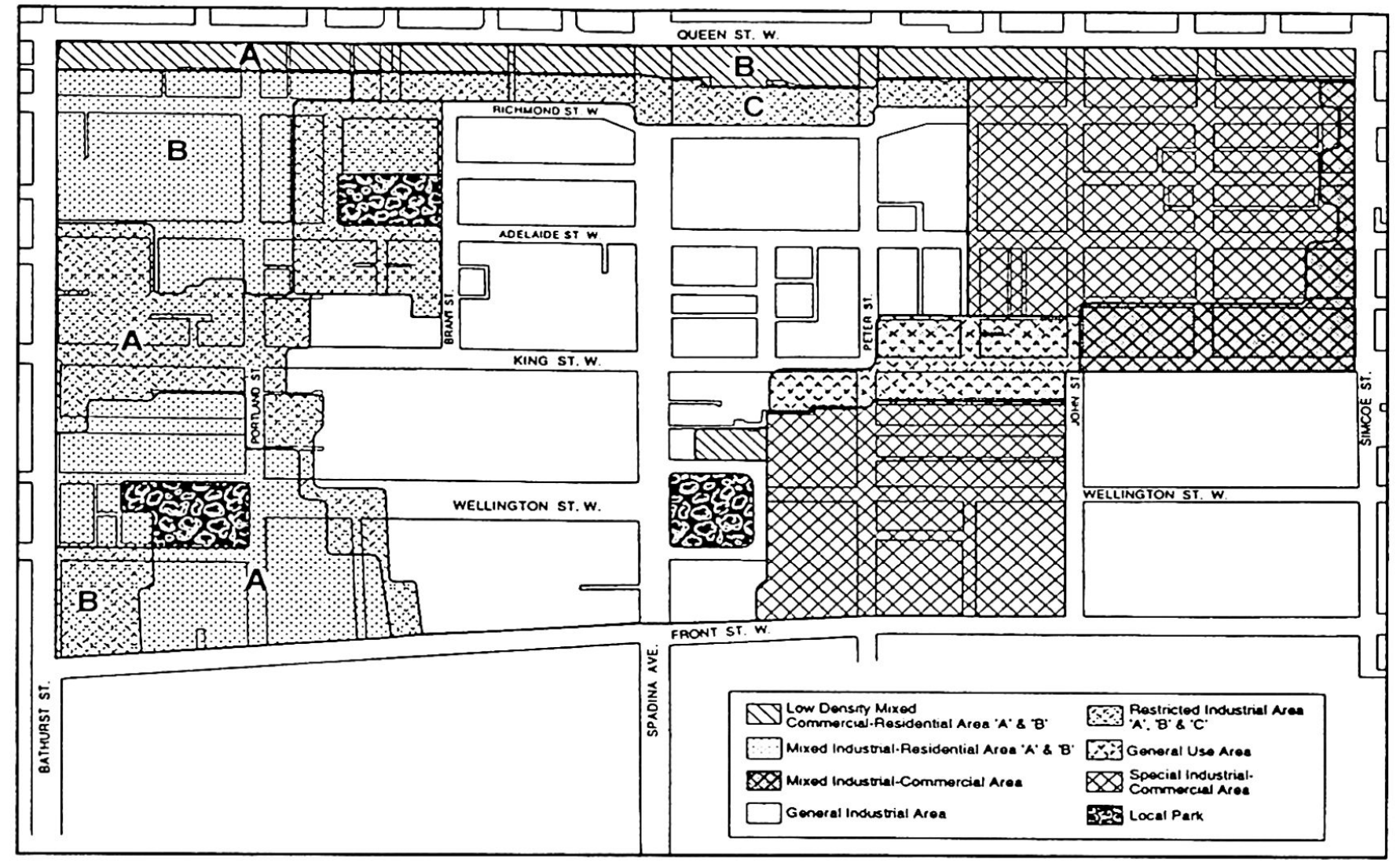

Fig. 3: Nouveau plan directeur: King-Spadina Official Part II (1988)

New general plan: King-Spadina Official Plan Part II of 1988

Neuer Leitplan: Offizieller Entwicklungsplan Teil II für King-Spadina, 1988

Source: CiTY OF TORONTO (1996: 24) 
King-Spadina à l'ouest et King-Parliament à l'est. Cette pression se manifeste notamment par une augmentation des prix fonciers qui rend financièrement impossible la rénovation ou la construction d'un nouveau bâtiment qui serait dédié uniquement à une utilisation industrielle. Il n'est alors pas rare que le propriétaire choisisse de démolir un vieil immeuble et d'y aménager des places de stationnement sur le terrain vacant afin d'obtenir le meilleur rendement possible compte tenu des règles d'urbanisme en vigueur. Mais il est évident que ce procédé est temporaire. Il signale une anticipation des propriétaires qui souhaitent restructurer le quartier soit pour y étendre la $\mathrm{CBD}$, soit pour y réaliser des magasins et des appartements de luxe (KING 1988). Ils se préparent déjà à tirer profit d'un futur changement du régime de l'affectation du sol en veillant justement à créer ou à participer à l'émergence des conditions nécessaires à ce changement.

\section{Adaptations du régime de l'affection du sol}

Les altérations qui se produisent dans l'environnement de King-Spadina placent les autorités locales dans un contexte particulièrement difficile. Elles doivent en effet concilier:

- un discours politique qu'elles dédient à la préservation du caractère industriel du quartier et au maintien d'une forte diversité des emplois offerts dans le centre de la ville;

- les intérêts de l'industrie de la mode en particulier dont elles ont besoin pour fonder et justifier leur engagement politique;

- leur désir de préserver un patrimoine historique et architectural.

Ces exigences ne sont pas exemptes d'ambiguïtés et de contradictions. Elles vont en fait se traduire par une politique, développée sous l'égide du service de l'urbanisme, qui va s'assouplir graduellement. Les adaptations de la régulation recouvrent deux formes principales. L'une est liée à des modifications du zonage (plan directeur de quartier et plan d'affectation). L'autre, nommée site specific agreement, relève davantage d'opportunités offertes par des opérations de partenariat public-privé. Elle consiste à appliquer un traitement dérogatoire à des projets spécifiques. Trois exemples sont présentés dans les lignes qui suivent. Ils sont agencés selon un ordre chronologique. Le premier et le troisième procèdent de la première forme (évolution du zonage), tandis que le second est caractéristique de la deuxième forme (site specific agreement).

\subsection{Altérations du zonage}

(jusqu'à fin des années quatre-vingt-dix)

En 1957, le plan de zones initial prévoit que la majeure partie du quartier soit affectée à l'industrie. De nombreuses activités commerciales y sont cependant autorisées, tandis que la fonction résidentielle est proscrite. En 1973, une association de résidents habitant des maisons situées dans le secteur ouest de King-Spadina demande aux autorités de régulariser leur situation. Le service de l'urbanisme profite de cette requête pour lancer une étude plus globale qui débouche en 1978 sur l'approbation du plan directeur de quartier nommé King-Spadina Official Plan Part II. A ce moment, les aménagistes sont surtout préoccupés par une extension possible des commerces et des bureaux qui s'opérerait à l'est, au détriment de l'industrie. Le contenu du plan directeur est empreint de cette inquiétude. Ce document confirme la prédominance de l'affectation industrielle. Mais il autorise désormais la fonction résidentielle dans le secteur ouest et crée surtout une zone tampon à l'est dédiée à des espaces commerciaux ou de bureaux. Par ailleurs, la définition même de l'affectation industrielle est assouplie. Dans la zone nommée general industrial area, le quart de la surface brute de plancher peut être consacré à des commerces. Cette mesure est offerte à titre d'incitation. Il s'agit d'encourager les propriétaires à rénover les bâtiments industriels traditionnels qui constituent désormais un patrimoine digne de protection. Quant aux showrooms nécessaires à l'industrie de la mode, aux back offices des grandes entreprises tertiaires localisées dans la CBD, aux imprimeries ou aux entreprises consacrées au cinéma, à la photo et au graphisme, ils viennent dorénavant compléter la liste des utilisations autorisées en zone industrielle.

En 1980, le plan directeur fait l'objet d'une première révision. L'objectif des aménagistes ne varie pas. Il s'agit toujours de contenir les stratégies anticipatrices des propriétaires dont les biens sont situés à la marge de la $\mathrm{CBD}$. Pour réduire leurs spéculations qui se matérialisent par un désinvestissement dans l'environnement construit et par une augmentation du nombre de transactions foncières, les autorités préconisent la création de deux nouvelles zones: general use area et special industrial-commercial area. Les bureaux et les commerces peuvent désormais y occuper la moitié de la surface brute de plancher. Elles espèrent, avec ces nouvelles mesures, restaurer l'intérêt des propriétaires à entreprendre dans le quartier. Cette version du plan directeur reste en vigueur jusqu'en 1988.

Pendant ces huit années où la régulation générale semble stable, diverses propositions émergent ce- 
pendant. Leur réceptivité auprès des aménagistes de la ville de Toronto demeure liée à la crainte de ces derniers de ne pouvoir maîtriser la pression que la CBD exerce sur l'ensemble du quartier KingSpadina. Certaines mesures visent à augmenter l'efficacité de la zone tampon. Elles débouchent sur la création d'un secteur autorisant les grands restaurants et les établissements liés à la vie nocturne (entertainment district). Elles reviennent à accroître les zones nommées general use area et special industrial-commercial area et à augmenter, une fois de plus, la liste des utilisations permises en zone industrielle. Elles impliquent donc une modification du plan directeur qui est entérinée en 1988 (fig. 2). D'autres en revanche s'appuient justement sur des formules de partenariat public-privé.

\subsection{Accords fondés sur le partenariat public-privé (années quatre-vingts)}

La négociation, au cas par cas, de projets non conformes à la réglementation en vigueur mais qui sont susceptibles de soutenir les industries localisées dans le quartier, caractérise cette approche dont la réalisation du Fashion Trade Center offre un exemple tout à fait significatif.
L'Ontario Fashion Exhibitors (OFE), une association qui organise des salons de la mode, est à l'origine du Fashion Trade Center. A l'étroit dans les locaux qu'elle occupe à King-Spadina et faute de trouver une alternative à des prix abordables dans le quartier, cette organisation envisage de déménager dans un site proche de l'aéroport, à quelques trente kilomètres du quartier traditionnel de KingSpadina. Un tel déménagement est perçu comme étant extrêmement dommageable pour l'industrie de la mode. Sachant que la construction de nouveaux locaux pour l'OFE n'est financièrement pas viable, ses représentants et le service de l'urbanisme de la ville tentent alors d'intéresser un partenaire privé afin de trouver une solution à l'intérieur du quartier King-Spadina.

IDJ, un promoteur immobilier, entre en scène en 1986. Il est prêt à offrir des espaces d'exposition à l'OFE pour autant qu'il obtienne l'autorisation de construire un hôtel. IDJ justifie cet équipement pour héberger les futurs clients des salons de l'OFE. Cette proposition est vigoureusement soutenue par les représentants de l'industrie de la mode. Elle obtient rapidement l'aval du service de l'urbanisme qui estime que l'autorisation d'un

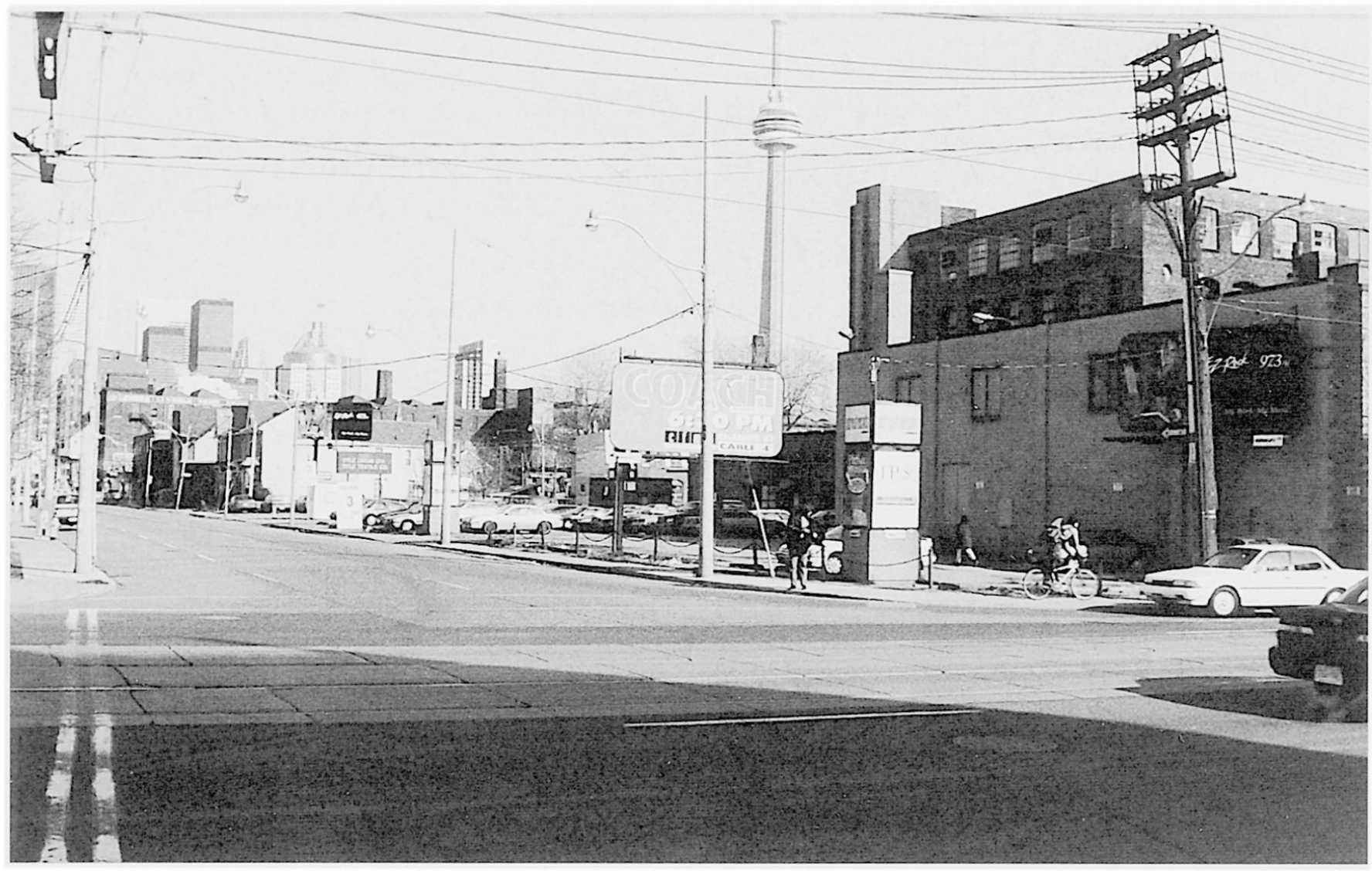

Photo 2: King-Spadina: un potentiel de reconversion qui est considérable

King-Spadina, an area of considerable redevelopment potential

King-Spadina, ein Gebiet mit vielversprechendem Aufwertungspotential

J. RUEGG, février 1997 
hôtel en pleine zone industrielle est parfaitement défendable si elle permet de maintenir les salons de l'OFE dans le quartier King-Spadina. A la fin du printemps 1987, IDJ obtient les autorisations nécessaires pour réaliser un bâtiment qu'il nomme Fashion Trade Center. Les travaux débutent en février 1988 et sont terminés à la fin de 1990.

L'arrangement passé entre IDJ et la ville de Toronto constitue un exemple typique de site specific agreement. Le projet n'est pas conforme à la réglementation en vigueur. Il nécessite l'approbation d'un plan spécial qui autorise:

- la réalisation d'un hôtel (affectation non conforme à la réglementation de la zone industrielle);

- un accroissement de la densité (le promoteur demande un indice d'utilisation de 11 alors que le règlement prévoit 7 au maximum).

Dans cet exemple précis, les autorités de Toronto monnayent leur pouvoir de mise en œuvre du zonage contre la fourniture, par le privé, de facilités qui participent à soutenir une industrie fragile. En l'occurrence, elles utilisent le projet d'un promoteur comme instrument de réalisation de leur propre politique industrielle.

Bien que le système juridique ontarien ne reconnaisse pas le précédent, il est évident que les site specific agreements confortent dans leur attitude ceux qui spéculent sur un changement complet des règles en vigueur dans le quartier. Ces partenariats influencent la gestion de l'ensemble du quartier de King-Spadina, même si leurs effets pour soutenir l'industrie sont globalement modestes. Dans les années quatre-vingt-dix, les autorités locales développent alors de nouvelles propositions, toujours dans le souci de revitaliser le quartier.

\subsection{Dernières altérations au zonage (années quatre-vingt-dix)}

Avec la crise économique du début des années quatre-vingt-dix, la pression exercée par la CBD sur King-Spadina s'atténue. Parallèlement, le nombre des établissements et des emplois industriels poursuit son déclin. De nombreux bâtiments sont vides. Les spécialistes considèrent que le quartier offre un potentiel largement sous-exploité en termes de qualité de l'environnement construit, d'infrastructures existantes et d'accessibilité au centre de Toronto.

Pour eux, cette situation provient principalement de la politique précédente qui privilégiait trop la ségrégation des fonctions. Ils recommandent, par conséquent, la suppression de la régulation des usages du sol et proposent de la remplacer par une police des constructions plus détaillées réglemen- tant le volume et la hauteur des constructions, l'implantation et le rapport aux espaces publics. La politique qui mettait l'accent sur l'affectation du sol et qui tentait d'influencer le contenu même des relations sociales, économiques et territoriales au sein du quartier est alors remplacée par un régime qui privilégie l'urban design et la forme urbaine. L'essentiel du quartier est désormais placé sous l'égide de la Reinvestment area (fig. 3). Adoptée au printemps 1996, cette dernière stratégie devrait permettre de rénover des structures industrielles et d'y réaliser la mixité de fonctions: appartements, magasins, bureaux, ateliers d'artistes, voire locaux industriels (photo 1) (Miller 1996).

Cette évolution s'inscrit parfaitement dans la ligne des effets induits par les premières adaptations décidées à la fin des années soixante-dix. Déjà en 1989, il était possible d'observer que le marché foncier était actif et que les dispositions retenues par le service de l'urbanisme ne parvenaient pas à le modérer. Entre 1976 et 1988, même si l'environnement construit a peu changé, ce sont environ $60 \%$ des 700 propriétés situées dans la moitié nord du quartier qui ont changé de main au moins une fois. Ces échanges, qui se sont traduits par une augmentation des prix fonciers, constituaient déjà une force inébranlable qui préfigurait les changements intervenus en 1996.

"Although, the fashion industry is not threatened directly, the pressure exerted by speculators and others on surrounding properties could become the major force to entirely modify the industrial environment of King-Spadina" (RUEGG 1989: 32).

\section{Remarques finales}

Les trois altérations majeures présentées ci-dessus posent alors bien le cadre dans lequel il y a lieu de s'interroger sur l'efficacité des règles d'urbanisme. Ces remarques finales ne prétendent évidemment pas faire le tour de cette question difficile. Elles doivent plutôt être prises comme des invites au débat. Elles sont organisées autour de deux idées: les limites des pratiques négociées et les limites de politiques fondées sur l'affectation du sol mais déconnectées de la question foncière. Pour simplifier aussi le cadre de l'analyse, le postulat selon lequel les autorités ont toujours légitimement et sincèrement œuvré en faveur de la défense de l'utilisation industrielle du quartier n'est pas remis en question. Il mériterait pourtant d'être soumis à l'analyse critique, puisqu'il n'est pas possible d'exclure à ce stade l'hypothèse selon laquelle la sauvegarde de la forme architecturale constituait en fait l'objectif qu'elle poursuivait réellement. 


\subsection{Limites aux pratiques négociés}

Dans le cas du Fashion Trade Center, les modalités de la négociation engagée par les autorités souffrent de plusieurs critiques. La négociation est menée en dehors de tout contrôle démocratique, entre le promoteur et les services compétents de la ville de Toronto. Il faut relever en outre que ces derniers opèrent sans avoir préalablement fixé avec l'autorité politique ni les limites à la négociation, ni le type de contrepartie qu'il s'agit d'obtenir. Ainsi, IDJ obtient finalement des avantages supérieurs à ceux qu'un promoteur peut escompter dans la $\mathrm{CBD}$, laquelle fait l'objet d'une application plus stricte des règlements en vigueur.

Et puis surtout, les autorités ne sont pas parvenues à assurer la durabilité de leur arrangement avec IDJ. Le bâtiment nommé à l'époque Fashion Trade Center n'abrite aujourd'hui plus aucune surface en faveur de l'OFE. En revanche, les entreprises de la mode disposent d'un nouvel immeuble situé un bloc plus au nord.

Un projet comme le Fashion Trade Center oblige alors à s'interroger sur la finalité des pratiques négociées et sur les garde-fous qu'il est nécessaire d'établir pour éviter qu'elles ne soient contreproductives et qu'elles ne finissent, dans le cas précis, par mettre en péril l'industrie de la mode. Quand des formules partenariales sont retenues, il faut veiller à définir un cadre strict à la négociation (Ruegg, Metran \& Vodoz 1992). Ce dernier pourrait par exemple prendre la forme d'un cahier des charges préalablement approuvé par les autorités compétentes (ZUPPINGER 1991).

\subsection{Limites à l'adaptation du régime de l'affection du sol}

Les motivations des autorités sont compréhensibles. Elles modifient le régime de l'affectation du sol et elles élargissent la définition même des usages industriels afin de rester en phase avec les évolutions du fonctionnement et des besoins des entreprises sises à King-Spadina. Mais la question demeure de savoir jusqu'où il est possible d'altérer la régulation sans aller trop loin dans un domaine où la difficulté majeure consiste à anticiper correctement et à définir un référentiel temporel pertinent. La capacité régulatrice de l'acteur public est ainsi souvent mise à mal lorsqu'il est contraint d'agir réactivement, par des mesures qui s'imposent seulement dans le court terme. Avec les derniers développements intervenus en 1996, l'objectif du maintien de l'industrie à King-Spadina ne peut pratiquement plus être satisfait par les autorités locales. Il dépend du seul marché. Les barrières visant à protéger les usages industriels ont en effet disparu. Certes, les consultants estiment que les tentatives précédentes pour défendre le secteur secondaire ne sont pas remises en question (MILLER 1996). Mais ils basent leur affirmation sur le constat d'un marché trop déprimé pour engendrer une pression qui menacerait les entreprises industrielles encore présentes à KingSpadina. Autant dire que si l'activité immobilière sort de son marasme, ces dernières en seront les premières victimes. Elles ne résisteront pas longtemps à une nouvelle hausse des loyers.

L'évolution de la régulation à King-Spadina s'inscrit alors dans le courant désormais bien connu de la dérégulation. Cette façon de composer avec une flexibilité rendue nécessaire par les anticipations des utilisateurs du sol laisse songeur. Ne va-t-elle pas d'abord dans le sens des intérêts dominants (BURGESs 1994) et ne souligne-t-elle pas ensuite la faiblesse des dispositifs d'urbanisme qui ne traitent pas de front la question foncière? Le zonage ne peut en effet exercer son effet stabilisateur sur les prix fonciers que s'il est mis en œuvre avec fermeté, dans la durée. Or cette exigence est en totale contradiction avec le développement de notre société qui ne cesse de réclamer la souplesse. A ce jeu, la situation de King-Spadina démontre la fragilité du partenaire public qui se trouve écartelé. Faute d'être propriétaire ou de mener une politique foncière, il se voit finalement dicter sa conduite par le marché. Mais, en posant qu'une régulation territoriale est indispensable (RoweIs 1983) et qu'elle doit composer avec l'institution de la propriété privée du sol (Ruegg 1996), ce constat est inacceptable. En modifiant continuellement les règles d'urbanisme, en supprimant largement les exigences liées à l'affectation et en s'en remettant à la taille considérable du quartier (photo 2) et à un marché qui peine à retrouver ses marques pour promouvoir une diversité des utilisations du sol, le risque est bien trop grand pour l'acteur public de perdre toute maîtrise sur le développement urbain et de se retrouver confiner dans un débat sur la forme et l'urban design.

L'exemple de Toronto rappelle alors que l'acteur public se doit d'appliquer les instruments de l'urbanisme dans un esprit rigoureux, créatif et visionnaire, s'il entend moduler à moyen et long terme les excès liés au fonctionnement du marché et agir sur la fabrique de la ville.

\section{Bibliographie}

Воотн, P. (1989): How Effective is Zoning in the Control of Development. Environment and Planning B: Planning and Design 16: 401-415.

BuRgess, P. (1994): Planning fot the Private Interest: Land Use Controls and Residential Patterns in Columbus, Ohio, 1900-1970. Columbus, OH: Ohio State University Press. 
Christman, J. (1994): The Myth of Property : toward an Egalitarian Theory of Ownership. New York: Oxford University Press.

City OF Toronto (1996): King-Spadina: Official Plan Part II.Toronto: City of Toronto, Planning and Development.

Frisken, F. (1988): City Policy-Making in Theory and Practice: the Case of Toronto's Downtown Plan. London, Ont: The University of Western Ontario. Local Governement Case Studies 3. (1992): Garreau, J. Edge city: Life on the new frontier. New York.

GiBBS, D. (1988): Restructuring in the Manchester Clothing Industry: Technical Change and Interrelationship between Manufacturers and Retailers. Environment and Planning A 20: 1219-1233.

GreENBERG, K. \& F. LewINBERG (1996): Reinventing Planning in Toronto. Plan Canada. May: 26-27.

KING, J. (1988): Protecting Industry from Yuppies and other Invaders. Planning. June: 4-8.

Miller, G. (1996): Toronto Considers Downtown Zoning Change. Planning. March: 26-27.

RoweIS, S. (1983): Urban Planning as Professional Mediation of Territorial Politics. Environment and Planning D: Society and Space 1: 139-162.

RUEGG, J. (1997): Origine, portée et enjeux du zonage: propriétaires en zone à bâtir. Thèse de doctorat présentée à la faculté des Sciences politiques et sociales de l'Université de Lausanne. Lausanne: Repro.

RUEGG, J. (1996): Champ du management territorial. Dans: Le management territorial, édité par DECOUtere, S., RuegG, J. \& D. Joye: 11-24. Lausanne: Presses polytechniques et universitaires romandes. RuegG, J. (1992): Fashion Trade Center à Toronto. Dans: La négociation, édité par J. RuEGG, N. METTAN, ET L. VoDoz: 128-134. Lausanne: Presses polytechniques et universitaires romandes.

RuegG, J., MetTan, N. \& L. Vodoz (1992): La négociation: son rôle, sa place dans l'aménagement du territoire et la protection de l'environnement. Lausanne: PPUR.

RuegG, J. (1989): Planning Measures... Do they really Participate in Sustaining the Fashion Industry in King-Spadina? Current Issue Paper, Department of Geography, University of Toronto (polycopié).

ScotT, A. (1988): Metropolis: From the Division of Labor to the Urban Form. Berkeley: University of California Press.

ScotT, A. (1984): Industrial Organization and the Logic of Intra-Metropolitan Location: a Case Study of the Women's Dress Industry in the Greater Los Angeles Region. Economic Geography 60, 1:3-27. ZuPPINGER, U. (1991): Vers un nouveau type de règlement du plan d'affectation communal. Liebefeld: PNR-Sol; publication 46.

\section{Résumé: Les règles d'urbanisme servent-elles le développement urbain? Réflexions à partir d'un exemple canadien}

Cet article offre une discussion sur les liens qui relient la fabrique de la ville aux règles d'urbanisme. Il s'agit plus précisément d'examiner si ces règles influencent les projets des particuliers, si elles permettent de gérer les externalités qu'ils génèrent et de les coordonner ou si, au contraire, elles sont inopérantes face aux exigences des promoteurs et des agents de la construction. Dans un régime où la propriété privée du sol n'est absolument pas remise en question et dans un contexte spécifique où des phénomènes puissants de déterritorialisation et de reterritorialisation sont à l'œuvre, les dispositifs traditionnels fondés sur le zonage (land use planning) sont largement insuffisants. Mais cette affirmation s'appuie ici sur le seul exemple du quartier industriel de King-Spadina à Toronto. Or il s'agit d'un cas presque caricatural où pratiquement toutes les utilisations du sol sont susceptibles de changer rapidement. Il ne permet donc pas de tirer des enseignements généraux et définitifs. Mais il est parfaitement adéquat pour lancer le débat et s'interroger sur les modalités de l'efficacité de l'action planificatrice et sur ses liens avec l'institution de la propriété privée du sol.

\section{Summary: Do planning measures aid the urban process? A discussion based on a Canadian example}

This article is an attempt to assess how planning measures interplay with the urban process. Do they help to better coordinate private projects, and to better manage the various externalities that are linked to the urban fabric? Or do planning measures rather seem incapable of dealing with developers' requirements? In situations where private landownership is not called into question and where powerful forces are modifying territorial relations, land use planning regulations are not sufficient. This argument, however, is based only on the industrial area of King-Spadina in Toronto which is a very specific case, almost caricatural, because land use is under strong pressure for major changes. Therefore it does not allow to draw many lessons from it. Nevertheless, it is perfectly suitable to introduce an interesting debate, to better understand the general conditions that are to be met to improve the efficiency of planning measures, and to better judge their links with the private landownership institution. 


\section{Zusammenfassung: Dienen Stadtplanungs- bestimmungen der Stadtentwicklung? Auf ein kanadisches Beispiel gestützte Überlegungen}

Der Beitrag stellt die Beziehungen zwischen Bestimmungen im Bereich der Stadtplanung und der Stadtentwicklung zur Diskussion. Es wird den Fragen nachgegangen, ob solche Bestimmungen eine Einflussnahme auf Projekte von privaten Trägern ermöglichen und einen gerechten Umgang mit den erzeugten Externalitäten sowie deren Koordination erlauben, oder, ob im Gegenteil, diese Bestimmungen gegenüber den Anforderungen der Bauträger wirkungslos sind. Es wird argumentiert, dass die traditionellen, auf Zonierungen gestützte Planungsvorkehrungen (land use planning) ungenügend sind, wenn es um ein System des unantastbaren privaten Grundbesitzes geht und wirkungsvollen Entterritorialisierungs- und Reterritorialisierungskräften ausgesetzt ist. Diese Überlegungen beruhen auf dem Fallbeispiel des KingSpadina-Industriequartiers in Toronto. Es weist beinahe karikaturenhafte Züge auf, praktisch alle Bodennutzungen können einem raschen Wandel unterzogen werden. Deshalb können keine allgemeingültigen oder abschliessenden Erkenntnisse abgeleitet werden. Der Fall eignet sich hingegen sehr gut zur Eröffnung einer Diskussion über die Wirkungsweise von planerischen Tätigkeiten und deren Verbindungen mit der Institution des Privatgrundbesitzes.

Jean Ruegg, Dr en Sciences sociales, MScPl, Communauté d'études pour l'aménagement du territoire (C.E.A.T.), Ecole Polytechnique Fédérale de Lausanne, 14 av. de l'Eglise Anglaise, CP 555, 1001 Lausanne; Institut de Géographie de l'Université de Fribourg, Pérolles, 1700 Fribourg. 
